\title{
Perceptual selectivity for color and form
}

\author{
JAN THEEUWES \\ TNO Institute for Perception, Soesterberg, The Netherlands
}

\begin{abstract}
Three visual-search experiments tested whether the preattentive parallel stage can selectively guide the attentive stage to a particular known-to-be-relevant target feature. Subjects searched multielement displays for a salient green circle that had a unique form when surrounded by green nontarget squares or had a unique color when surrounded by red nontarget circles. In the distractor conditions, a salient item in the other dimension was present as well. As an extension of earlier findings (Theeuwes, 1991), the results showed that complete top-down selectivity toward a particular feature was not possible, not even after extended and consistent practice. The results reveal that selectivity depends on the relative discriminability of the stimulus dimensions: the presence of an irrelevant item with a unique color interferes with parallel search for a unique form, and vice versa.
\end{abstract}

This article deals with the ability of the parallel stage of visual processing to selectively guide the subsequent serial employment of attention. The present approach presumes that visual-information processing consists of two functionally independent stages: an early, preattentive stage that operates without capacity limitations and in parallel across the visual field, followed by the later, limitedattentive-capacity stage that can deal with only one item (or a few items) at a time (e.g., Broadbent, 1958, 1982; Neisser, 1967; Treisman \& Gelade, 1980).

Theeuwes (1991) showed that the parallel stage cannot selectively guide the attentive stage to the task-relevant stimulus dimension: when color was relatively more discriminable than form, then the presence of an irrelevant item with a unique color interfered with parallel search for a unique form, and vice versa. It was concluded that during preattentive parallel search, subjects were not capable of selectively attending to just the known-to-berelevant stimulus dimension. Selectivity depended on the relative discriminability of the stimulus dimensions: when searching for an easily discriminated color (i.e., search for a red item among green nontarget items), the unique form did not interfere, while the unique form did interfere when the color discrimination was made much harder (i.e., a yellow/red target among yellow/green nontargets).

These findings suggested a model that assumes that the preattentive process computes, for each stimulus dimension, differences in features resulting in an activation map representing how different each item is from each of the other items within a particular feature dimension (e.g., color dimension, form dimension). This computation results in a difference signal at each location similar to the

Parts of this paper were presented at the 1991 meeting of the Psychonomic Society in San Francisco. I would like to thank Lester Krueger, Marvin Chun, Hal Pashler, and Bill Bacon for their excellent comments and suggestions. Correspondence concerning this article should be addressed to Jan Theeuwes, TNO Institute for Perception, P.O. Box 23, 3769 ZG Soesterberg, The Netherlands. bottom-up activation in the parallel stage of Cave and Wolfe's (1990) guided-search model. The parallel stage is then followed by a stage involving focal attention that is assumed to be directed serially and unintentionally to the location having the highest activity, the next highest, and so forth. Since it is assumed that these two stages operate independently of any strategic control, selectivity completely depends on the bottom-up activations caused by the differences in features within different stimulus dimensions. Similar to Sagi and Julesz (1985) and Ullman (1984), it is assumed that the parallel process can only perform a local-mismatch detection followed by a serial stage in which the most mismatching areas are selected for further analysis.

Although Theeuwes's (1991) findings did not lead to a definitive theoretical statement, the model above, describing selectivity solely in terms of bottom-up processing, seemed to account best for his data. Yet, many current theories assume that visual selection is affected by knowledge of the target to be found, implying that selection is sensitive to top-down processing. For example, the feature-integration theory (FIT; Treisman, 1988; Treisman \& Gelade, 1980) assumes at least selectivity between stimulus dimensions: subjects check for activity signaling a contrasting item in the relevant target-defining module and simply ignore the others. According to Cave and Wolfe's (1990) guided-search model, the bottom-up activations caused by the differences in features can be altered by top-down activity that depends on the knowledge of the target to be found, suggesting that the parallel stage can selectively guide focal attention to the likely target locations. Other models, such as those of Duncan and Humphreys (1989) and Bundesen (1990), also assume that preknowledge of the target to be found affects selectivity. Note, however, that all of these theories proposed a topdown component to account for conflicting data, with search for targets defined by conjunctions of elementary features. The present framework only regards selectivity 
between target and distractors when each is defined by a unique elementary feature.

In Theeuwes's (1991) experiments showing the absence of selectivity, subjects viewed multielement displays (5, 7 , or 9 elements) in which one item had a unique color while another item had a unique form. Different groups of subjects either searched for an item with a unique color or an item with a unique form. The results indicated that the item unique in the task-irrelevant dimension interfered with search for the item unique in the task-relevant dimension. It is important to note that subjects only knew which dimension was relevant. Thus, subjects searching for a unique color received blocks of trials in which a red item was located among green nontargets or a green item was located among red nontargets. Subjects searching for a unique form received blocks of trials in which a square was located among circles or a circle was located among squares. Thus, subjects did not exactly know the feature properties to attend to, that is, whether to search for a red or a green item or for a square or a circle. The same held for the distractor conditions: subjects knew that an item was present in an irrelevant dimension, yet they did not know the exact feature properties of the distractor. In addition, because target and nontarget display elements continuously switched roles, an automatic, consistently mapped (CM) detection response could not develop.

The question remains, then, whether selectivity can be obtained when not only the stimulus dimension is known (e.g., knowing that the target has a unique color), but also the exact feature value within that dimension (e.g., knowing that the target is green). Such a hypothesis is viable because in the previous study, uniqueness within a dimension was defined by the whole stimulus display; for example, within the color dimension, a green item was the target because the other items were red. In addition, to provide the most decisive test for the possibility of selectivity during preattentive parallel search, in the present study, subjects always searched for the same target feature (i.e., a green circle), which was never used as a nontarget. In line with Shiffrin and Schneider's (1977) analysis, this should lead to an automatic detection response of the target.

\section{EXPERIMENT 1A}

Subjects viewed multielement displays $(5,7$, or 9 elements) in which the target line segment that determined the correct response was always located in the green circle. In the no-distractor color condition, this green circle had a unique color because it was embedded among red circles. In the distractor condition, one of these red circles was a red square. In the no-distractor form condition, the green circle had a unique form because it was embedded among green squares. In the distractor condition, one of these green squares was red.

\section{Method}

Subjects. Sixteen right-handed subjects, ranging in age from 18 to 28 years, participated as paid volunteers. Eight subjects were randomly assigned to the form condition, and 8 to the color condi- tion. All had normal or corrected-to-normal vision and reported having no color-vision defects.

Apparatus. An S-R interface with external clocks (accurate to $1 \mathrm{msec}$ ) connected to an IBM AT-3 with video digitizer (Matrox) controlled the timing of the events, generated video pictures, and recorded reaction times (RTs). The response panel was tilted $45^{\circ}$, and consisted of left and right response keys $(1 \times 1 \mathrm{~cm})$ mounted $5.5 \mathrm{~cm}$ apart.

The stimuli appeared on a $35 \times 23 \mathrm{~cm}$ TV monitor (Conrac Model $7250 \mathrm{C19}$ ). The fixation point and the line segments were presented in white $\left(17.0 \mathrm{~cd} / \mathrm{m}^{2}\right)$ on a black background $\left(.40 \mathrm{~cd} / \mathrm{m}^{2}\right)$. The surrounds were either red or green (CIE $x, y$ chromaticity coordinates of $.622 / .357$ for red and $.282 / .596$ for green) and were matched for luminance $\left(5.5 \mathrm{~cd} / \mathrm{m}^{2}\right)$.

Each subject was tested in a sound-attenuated, dimly lit $2 \times 2 \times 2 \mathrm{~m}$ cubicle (Amplisilent) with his/her head resting on a chinrest adjusted to a comfortable height. The TV monitor was located at eye level, $118 \mathrm{~cm}$ from the chinrest. An intercom was used for communication with the subject.

Stimuli. The task was very similar to that in Theeuwes (1991). The stimulus field consisted of 5,7 , or 9 elements equally spaced around the fixation point on an imaginary circle whose radius was $3.4^{\circ}$. Display elements were outline circles $\left(1.4^{\circ}\right.$ of diameter) or squares $\left(1.4^{\circ}\right.$ on a side), each containing a line segment $\left(0.5^{\circ}\right)$ that was tilted $22.5^{\circ}$ to either side of the horizontal or vertical plane. These orientations were randomly distributed in a display. In each display, there was a single green circle that contained a line segment that was oriented either horizontally or vertically, the latter orientation determining the appropriate response key (left for vertical and right for horizontal). For the group of subjects in the form condition, the green circle containing the target line segment had a unique form, because it was surrounded by 4,6 , or 8 green squares. For the group of subjects in the color condition, the green circle containing the target line segment had a unique color because it was surrounded by 4,6 , or 8 red circles. Because the subjects responded to the orientation of a target line segment located among slightly tilted nontarget line segments, the task required focal attention (Theeuwes, 1991; Treisman \& Gormican, 1988), but not a high spatial acuity.

Procedure. The sequence of events was as follows: Initially, a white fixation dot $\left(.3^{\circ}\right)$ was presented at the center of the visual field. Six hundred milliseconds prior to display onset, the fixation dot increased in size to $2^{\circ}$ to warn the subject. The stimulus field remained present for a maximum of $4 \mathrm{sec}$ until a response was emitted.

The group of subjects in the form condition received two conditions: (1) a no-distractor condition, in which the green circle containing the target line segment was surrounded by 4,6 , or 8 green squares, and (2) a distractor condition, in which one of these green squares had a red color. The group of subjects in the color condition received two conditions as well: (1) a no-distractor condition, in which the green circle containing the target line segment was surrounded by 4,6 , or 8 red circles, and (2) a distractor condition, in which one of these red circles was a square. As will be clear, when searching for a unique form (form condition), the distracting element had a unique color, and when searching for a unique color (color condition), the distracting element had a unique form. The position of the green target circle in the circular display was randomized from trial to trial. Figure 1 shows the various display configurations.

There were two blocks of 144 trials each in the no-distractor condition and two blocks of 144 trials each in the distractor condition. Each subject performed a total of 288 trials in each distractor condition-that is, a total of 96 trials in each display-size condition. Half of the subjects started off with the no-distractor condition, and the other half with the distractor condition. Display size was randomized within blocks. The practice session consisted of 144 no-distractor and 144 distractor trials. 

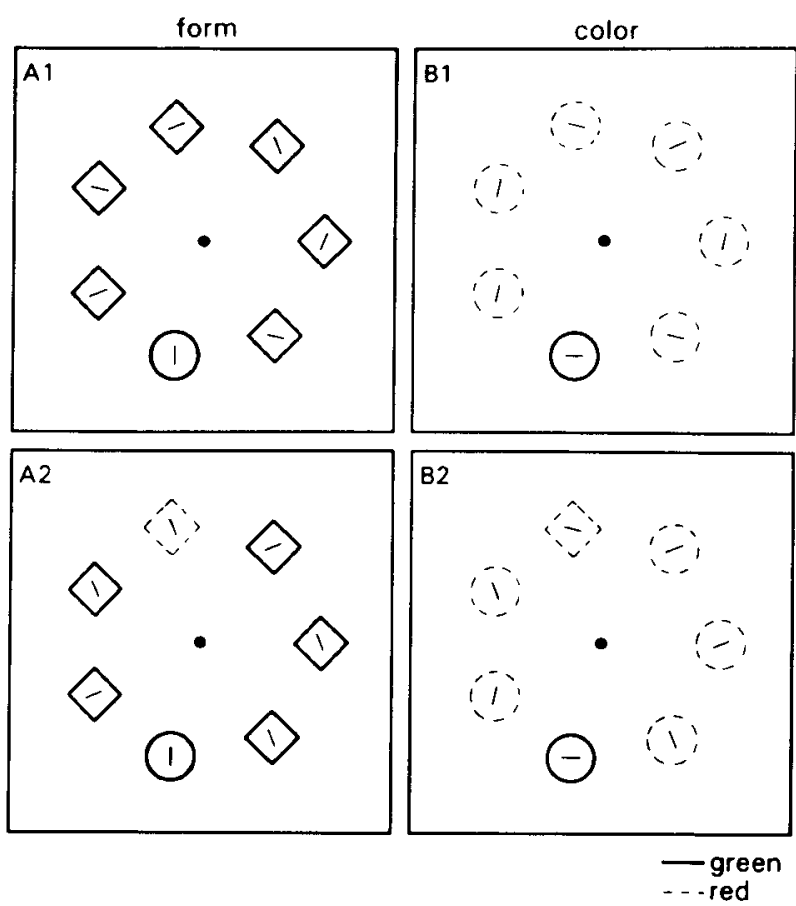

Figure 1. Examples of Display Size 7. The subjects always searched for a green circle surrounded by green squares (left side) or by red circles (right side), either without a distractor (top) or with a distractor item (bottom).

A session consisting of 144 trials lasted approximately $10 \mathrm{~min}$, with a 1-min break after 72 trials. Each block started with 3 dummy trials. Two subjects were run in alternating sessions. Prior to the start of the experiment, each subject was instructed to search for the horizontal or vertical target line segment and to press the appropriate response key with one of his/her thumbs, which were resting on the response keys. The subject was told that the line segment determining the response was always located in the green circle. It was emphasized that the subject should fixate the central dot and not move his/her eyes during the course of any trial. To ensure that the subject followed the instructions, during the practice session eye movements were monitored on line by means of an infrared camera. It was stressed that a steady fixation would reduce RT and make the task easier. Both speed and accuracy were emphasized. A warning beep informed the subject that an error had been committed. If no response was made after $4 \mathrm{sec}$, the subject was informed that he/she had committed an error. Before each session, the subject was informed about the upcoming condition.

\section{Results}

Response times longer than $1 \mathrm{sec}$ were counted as errors, which led to a loss of well under $1 \%$ of the trials. Figure 2 presents the subjects' mean RT and error percentages in the four conditions. For each of these measures, the form and color conditions were analyzed separately with display size and distractor condition as main factors.

In the form condition (Figure 2A), there was a main effect on RT for display size $[F(2,14)=8.2, p<.01]$ and for distractor $[F(1,7)=13.9, p<.01]$. In the color condition (Figure 2B), there was only an effect on RT for display size $[F(2,14)=14.3, p<.001]$. To determine the slopes of the RT functions, the individual mean RTs were submitted to a linear regression analysis. For the no-distractor and distractor conditions, the mean slopes for the form condition were 2.2 and $6.1 \mathrm{msec}$ and for the color condition, 5.2 and $4.4 \mathrm{msec}$. None of these slopes were significantly different from zero [all $t s(7)<1.63$ ], indicating parallel preattentive search across all items in the display. Inspection of the RT data reveals that search for the green circle when surrounded by green squares is distracted when one of the squares has a unique red color. The reverse does not hold: when searching for a green circle surrounded by red circles, the presence of a unique form does not affect search.

To achieve homogeneity of the error-rate variance, the mean error rates per cell were transformed by means of an arcsine transformation. For both form and color conditions, none of the error effects were significant, which suggests that the differences in RT are not due to trading speed for accuracy.

\section{Discussion}

The results of this experiment are quite similar to Theeuwes's (1991, Experiment 2) earlier findings: when searching for a target item that differs from the other elements in color, the presence of an element with a unique form had essentially no effect. Yet, search for a target item that differs from nontarget elements only in form is slowed down by the presence of a unique color.

The results indicate that knowing the exact target feature (i.e., the target is green and a circle) and knowing the exact distractor feature (i.e., a red square), both consistently mapped throughout the whole experiment, did

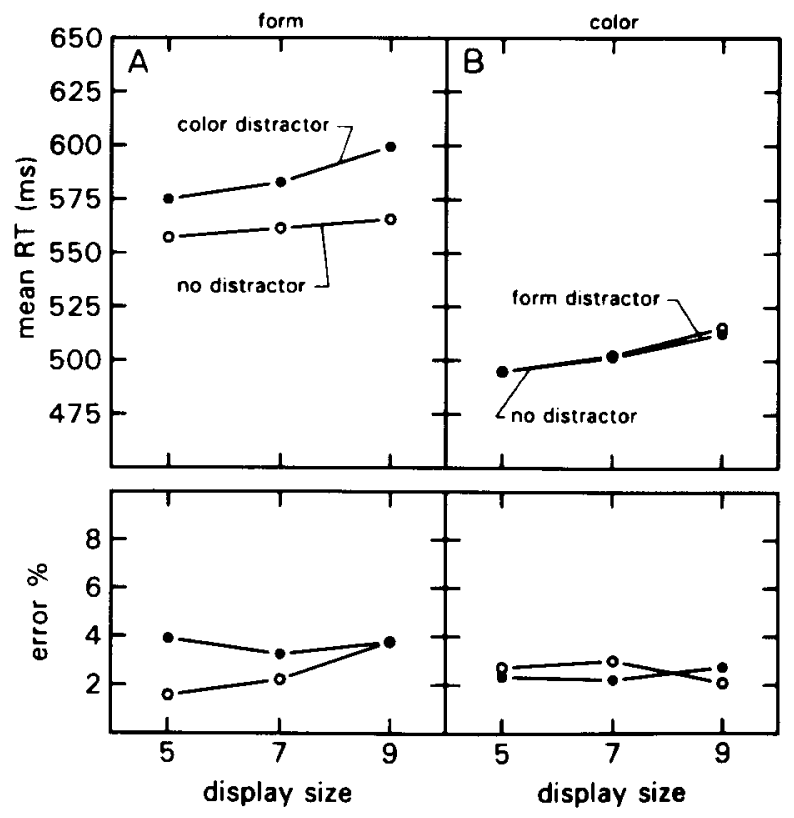

Figure 2. Experiment 1A: Mean reaction time and error percentages for search with or without a distractor for the form (Panel A) and color (Panel B) conditions. 
not result in top-down selectivity; when searching for a green circle surrounded by green squares, the presence of a red square clearly interfered.

The results are in line with the earlier outlined model, which assumes that selectivity primarily depends on bottom-up processing. According to this model, focal attention is unselectively captured by the first feature made available by the preattentive stage. In the present experiment, the subjects searched in both the form and color conditions for exactly the same stimulus (i.e., a green circle), which was either surrounded by green squares (form condition) or by red circles (color condition). Yet, the no-distractor conditions reveal that finding a green circle between red circles is about $60 \mathrm{msec}$ faster $[F(1,14)=$ $10.3, p<.01]$ than finding the same stimulus surrounded by green squares. This implies that the bottom-up activation of a particular target element depends on the feature properties of the surrounding elements: the green circle among red circles produces a much higher activation than does the green circle among green squares. This implies that the difference in color becomes available earlier in time than the difference in form, suggesting that in the form condition, attention is first captured by the uniquely colored distractor and then captured by the uniquely shaped target. This accounts for the observed asymmetric selectivity and explains why the color distractor only has an effect on the intercept and not on the slope of the search function.

Although the pattern of interference in the present experiment is equivalent to that in Theeuwes (1991, Experiment 2), it should be noted that the absolute RTs in the present experiment are much smaller than those reported in Theeuwes's experiment. In Theeuwes (1991), subjects searched for an item unique within a dimension (e.g., a red among green items or a green among red items), suggesting that target and nontarget display elements continuously switched from trial to trial. In the present experiment, target and nontargets never changed roles; that is, the subjects always searched for a green circle. Although one might suggest that the relatively low absolute RT levels in the present experiment reflect some top-down processing at the preattentive level, it is more conceivable that the RTs are fast because there is less variation within the task. The consistency within the task speeds up processes following the preattentive extraction of target and distractor items. For example, when focal attention is erroneously switched to the red square, deciding that the red square is not the target might be fast because the subjects consistently search for a green circle. Similarly, when focal attention is switched to the green circle, the subjects might be fast in deciding that what is selected is in fact the target because they consistently searched for it. Note, however, that these decisional processes operate on target and distractor items after they have been selected for further processing.

\section{EXPERIMENT 1B}

Experiment 1A showed that selectivity for the green circle primarily depends on the bottom-up activation caused by the surrounding elements. Selectivity could not be obtained by top-down processing: knowing the exact feature properties and the consistent mapping of targets and nontarget attributes did not result in selectivity. These findings are at odds with many models suggesting that selectivity is affected by top-down processing (e.g., Cave \& Wolfe, 1990; Duncan \& Humphreys, 1989; Treisman, 1988).

Reasons for not obtaining selectivity might be that 576 experimental and 288 practice trials are not enough to induce top-down selectivity, although usually in this type of task subjects receive many fewer trials. Experiment $1 B$ tested whether consistent and extended practice would result in the development of top-down control, that is, whether the irrelevant uniquely colored item would cease to interfere with parallel search for the unique form. In Experiment 1B, each subject received 288 practice trials followed by 1,728 experimental trials.

\section{Method}

Subjects. Eight subjects, ranging in age between 19 and 25 years, participated in the experiment.

Apparatus. The apparatus was identical to that in Experiment 1A. The display elements were either green or red (same CIE $x, y$ chromaticity as in Experiment $1 \mathrm{~A}$ ) and had a luminance of $6.8 \mathrm{~cd} / \mathrm{m}^{2}$.

Procedure. The task was identical to that in Experiment 1A, except that only the form condition was used, that is, the subjects searched only for a green circle among green squares. In the nodistractor condition, all squares were green, whereas in the distractor condition, one of the squares was red. The subjects were run in alternating blocks of 144 trials, which involved trials of either the no-distractor or the distractor condition. Half of the subjects had a no-distractor block first, and the other half had a distractor block first. Each subject received 144 no-distractor and 144 distractor practice trials, followed by a total of six blocks of trials in the no-distractor condition and six blocks of trials in the distractor condition.

\section{Results}

Response times longer than $1 \mathrm{sec}$ were counted as errors, which led to a loss of well under $1 \%$ of the trials. Mean RT and error percentages, divided into three blocks of 576 trials, are shown in Figure 3.

The individual mean RTs were analyzed with practice (Section 1, 2, 3), display size $(5,7,9)$, and distractor as factors. There was a main effect on RT for both display size $[F(2,14)=18.6, p<.001]$ and distractor $[F(1,7)=$ $14.4, p<.01]$. Note that there was no main effect on RT for practice and practice did not interact with any of the other variables, suggesting that consistent practice did not change any of the effects. For the no-distractor condition, slopes were $3.2,4.5$, and $6.0 \mathrm{msec}$ for Sections 1-3. For the distractor condition, these measures were $5.9,5.2$, and $7.0 \mathrm{msec}$. Only the latter slope differed significantly from zero $[t(7)=2.1, p<.05]$. The overall 


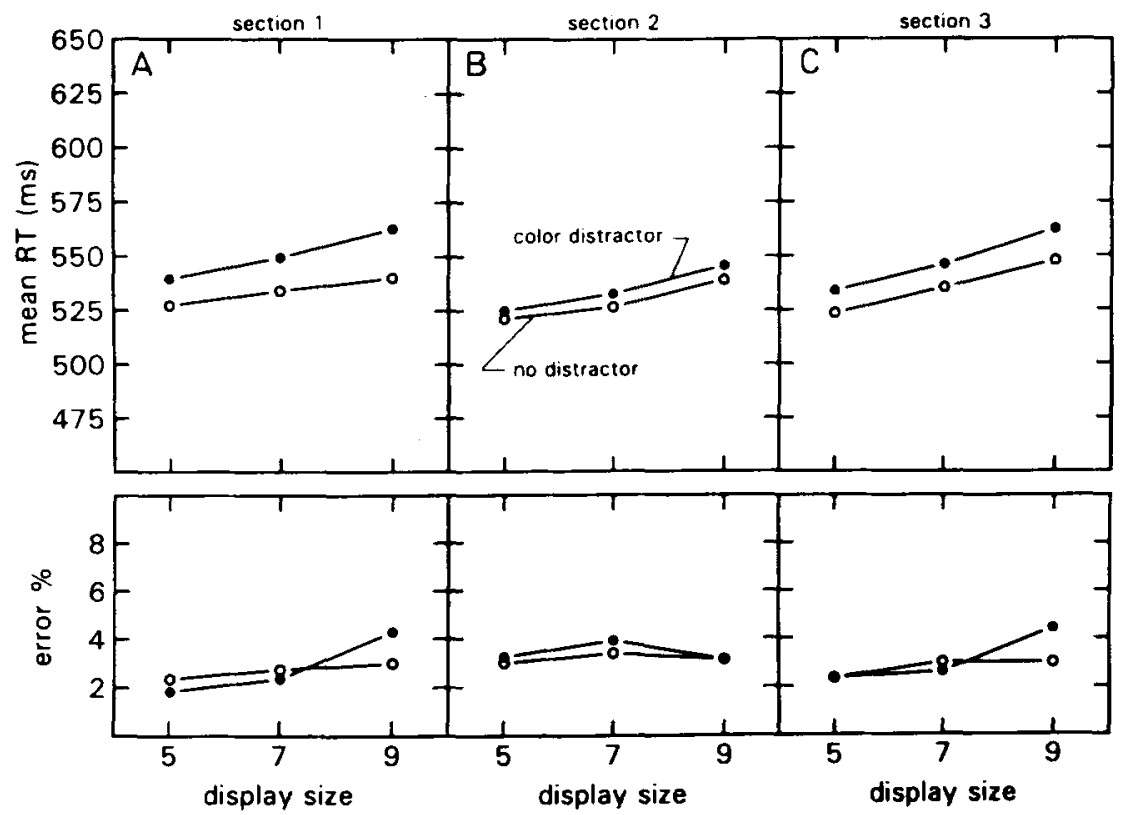

Figure 3. Experiment 1B: Mean reaction time and error percentages for search with or without a distractor separated in three levels of practice.

analyses suggest, as is evident in Figure 3, that consistent practice does not lead to an overall $\mathrm{RT}$ reduction or to a significant change in the relations between the variables, although it appears that the distractor effect is somewhat reduced in Section 2.

The arcsine-transformed error data showed only a main effect for display size $[F(2,14)=4.7, p<.05]$. Because this analysis indicates that error differences are nonsignificant or tend to mimic RT, differences in response latencies are not due to a speed-accuracy trade-off.

\section{Discussion}

The results of this experiment are fairly clear and confirm the conjecture that, even after extensive practice, complete top-down control is not possible: even after extended practice, subjects lack the ability to simply ignore the known-to-be-irrelevant color. The finding that practice does not alter the overall RT level suggests that the lack of selectivity cannot be attributed to insufficient practice. Apparently, the task is so simple that the initial 288 practice trials are enough to completely master the search task at a level that does not change with practice.

\section{EXPERIMENT 2}

Experiment 2 tested whether differences in bottom-up activations within each dimension can account for the observed asymmetric selectivity. On the basis of the results of Theeuwes (1991, Experiment 3), the color discrimination was made harder than the form discrimination. If attention is switched in the order of the availability of the local feature, it is expected that the asymmetry will switch as well, suggesting that the item with a unique form and not the item with a unique color will interfere.

\section{Method}

Subjects. Sixteen subjects, ranging in age between 18 and 24 years, participated in the experiment. Eight subjects were randomly assigned to the form condition; 8 were assigned to the color condition. Apparatus. The apparatus was identical to that in Experiment 1A.

Procedure. The task was identical to that in Experiment 1A. On the Basis of Theeuwes (1991, Experiment 3), the colors were made so similar that it could be expected that color differences were less salient than form differences. The color CIE $x, y$ chromaticity coordinates were $.435 / .488$ for yellowish red and $.400 / .515$ for yellowish green and were matched for luminance $\left(6.2 \mathrm{~cd} / \mathrm{m}^{2}\right)$. Design and procedure were identical to those in Experiment IA.

\section{Results}

Response times longer than $1 \mathrm{sec}$ were counted as errors, which led to a loss of about $1.25 \%$ of the trials. Mean RT and error percentages are shown in Figure 4.

In the form condition (Figure 4A), none of the variables showed a significant effect on RT, suggesting that finding a green circle among green squares was not hindered by the presence of a display element with a slightly different color. In the color condition (Figure 4B), there were main effects on RT for display size $[F(2,14)=41.1$, $p<.001]$ and for distractor $[F(1,7)=9.1, p<.05]$, implying that search for a yellowish-green circle surrounded by circles of a slightly different color is slowed down by the presence of a unique form. The mean slopes for the form conditions were 3.5 and $1.4 \mathrm{msec}$ for the no-distractor and distractor conditions. For the color condition, these slopes were 8.7 and $12.9 \mathrm{msec}$. Only the latter slope differed significantly from zero $[t(7)=2.0$, 

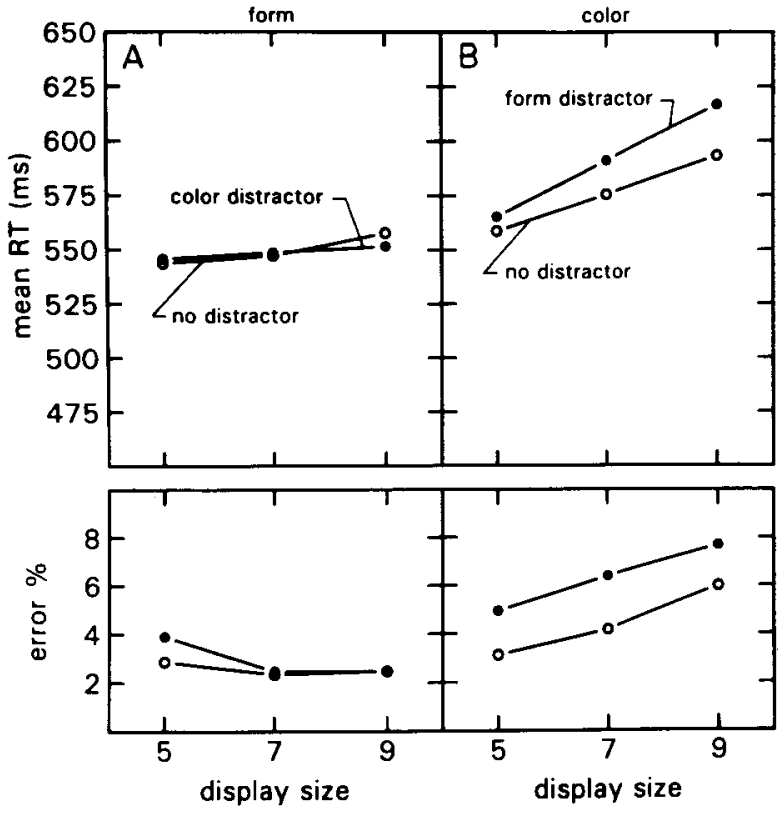

Figure 4. Experiment 2: Mean reaction time and error percentages for search with or without a distractor for the form (Panel A) and color (Panel B) conditions.

$p<.05]$, suggesting that in the color condition with a distractor, serial search starts to emerge. The other slopes did not differ from zero [all $t \mathrm{~s}(7)<1.43$ ], suggesting parallel search across all items.

The arcsine-transformed error data showed that in the form condition, none of the effects were significant, whereas in the color condition, there was a main effect for display size $[F(2,14)=10.3, p<.01]$. As this analysis indicates that error differences are nonsignificant or tend to mimic RT, differences in response latencies cannot be attributed to a speed-accuracy trade-off.

\section{Discussion}

The results clearly show that asymmetric selectivity, as observed in Experiment 1A, completely depends on the feature properties of the surrounding elements: if form is easier to discriminate than color, then search for form is not hindered by the presence of an element with a unique color, whereas search for color is affected by the unique form. Contrary to earlier findings (Theeuwes, 1991), the present experiment shows a complete reversal of the asymmetric selectivity between form and color. The results provide strong evidence for the earlier outlined model, which assumes that selectivity primarily depends on the bottomup activations caused by differences in features within stimulus dimensions. It assumes that focal attention is attracted to the location in the order of the availability of the difference signal at each location in the display. For example, attention may be attracted to a location of the difference signal as soon as the difference signal exceeds a particular threshold activation. Thus, focal attention is attracted to the location of the feature that "pops out" first, irrespective of whether that feature is a target or a distractor.

\section{GENERAL DISCUSSION}

The present experiments were designed to examine the extent to which the parallel preattentive stage can selectively guide the subsequent serial employment of attention. As the previous study (Theeuwes, 1991) had already indicated that during preattentive parallel search, complete selectivity toward a particular stimulus dimension does not occur, the present study extends these earlier findings and shows that complete selectivity toward a known-to-be-relevant stimulus feature does not occur either. In addition, complete selectivity cannot even be obtained after extended and consistent practice.

The present results are in line with the earlier outlined model, which assumes that the preattentive process calculates differences in features within dimensions, resulting in a pattern of activations at different locations, followed by an automatic shift of focal attention to the location of the feature that pops out first. Such a pop-out occurs irrespective of whether the popping-out feature is a target or a distractor. If the item that pops out first is the target, a response will be given; if it is not the target, attention will be unintentionally switched to the location having the next highest activation, and so on. The operation of the preattentive process is equivalent to the bottomup component of the Cave and Wolfe (1990) guidedsearch model. For example, the color activation at a particular location is calculated by finding the difference between the color at that location and the color at each of the other locations, and then combining all these color differences. Thus, when an item with a unique color and another item with a unique form are present simultaneously, the operation of the preattentive process gives rise to large difference signals at each of the locations of the odd items. Contrary to the claim of Cave and Wolfe (1990), the lack of selectivity as currently found might suggest that the parallel stage cannot identify anything and therefore cannot guide elements that are closest to the target value. In fact, the lack of selectivity suggests that the preattentive process has no access to the origins of the difference signals (e.g., whether they are caused by a unique color or a unique form). Therefore, knowing the exact target value (i.e., top-down effect) cannot affect the operations of the preattentive process because at the preattentive level, this information is not yet available. Only after entering the second stage of focal attention (i.e, after being selected) can knowledge regarding the target properties affect processing. Yet, these are top-down effects operating on items that have already been selected. Because it is assumed that the preattentive parallel stage is top-down impenetrable, subjects are not capable of activating the green target color or the circular target form (top-down activation of target values, as suggested by, e.g., Cave \& Wolfe, 1990) nor can they inhibit the square 
form distractor or the red color distractor (top-down inhibition of distractor values as suggested by, e.g., Treisman \& Sato, 1990). If the operations of the preattentive process are not under intentional control and are not sensitive to perceptual load (i.e., the absence of a displaysize effect), it can be argued that preattentive processing is strongly automatic because it satisfies both the load insensitivity and the unintentionality criterion of automaticity (see also Theeuwes, 1991).

Although the data-driven selection model as described above seems to account for the present data, it cannot be said that top-down control at the preattentive stage never occurs. It can be argued that in the present study, preattentive top-down control does not show up because the salient pop-out distractor generates a bottom-up activation that is so large that additional top-down activation (i.e., knowing the target and distractors) cannot completely prevent the distractor from being selected. Therefore, in displays in which there are no salient pop-out items, top-down processing may play a role. Note, however, that in displays without large bottom-up activations, as, for example, in displays with items consisting of conjunctions of features, preattentive parallel search does not occur at all: search is performed serially or partially serially through the display (e.g., the group-scanning hypothesis of Treisman \& Gormican, 1988). Thus, observed topdown effects with these types of tasks cannot be attributed to the preattentive parallel stage.

As an alternative interpretation of the present findings, one might consider a model of performance that assumes that the interference caused by the salient distractor $\alpha$ curs at the preattentive level rather than at the attentive level of processing. In this view, at the preattentive stage, subjects selectively attend to the target item; yet, the presence of the irrelevant distractor item slows down the preattentive processing of the target item. Although this model can account for the present findings, it assumes that color and form information interfere with each other at the preattentive level, a claim which is refuted by various studies showing that color and form information are registered independently and separately at the preattentive stage (e.g., Callaghan, Lasaga, \& Garner, 1986; Treisman \& Gelade, 1980).

Various recent theories of visual search assume topdown effects at the parallel stage of processing (e.g., Cave \& Wolfe, 1990; Duncan \& Humphreys, 1989; Treisman \& Sato, 1990) to account for relatively flat search functions when searching for targets defined by conjunctions of features. The present study involving feature search suggests that top-down control at the preattentive stage is absent, or at least so weak that it cannot prevent interference from the irrelevant distractor, not even after extended and consistent practice. Yet, there are various reasons why Theeuwes (1991) and the present study, unlike others, show interference from the nonrelevant dimension. First, because the interference effects are relatively small (about 15 to $25 \mathrm{msec}$ ), the addition of noise to the display will obscure the interference effect, especially be- cause the conclusion that there is no interference is reached by accepting a null effect. For example, noise is introduced when display elements are not presented in a regular pattern around the point of fixation but in matrix array. Especially with small display sizes, the matrix array may cause an irregular pattern with groups of clumped display elements. Pashler's (1988) texture-segregation studies reveal the difficulties with accepting a zero effect: Experiment 2 suggested that irrelevant color heterogeneity did not interfere with search for a particular form, whereas Experiment 4 (basically a replication) showed highly significant interference effects of the same color variation. Second, to disclose interference effects at the preattentive parallel level, it must be ensured that search is performed in parallel. If search is partially serial, as, for example, with conjunction search, then the effect of the distractor will be attenuated. Note that in texture-segregation studies investigating the interference of irrelevant dimensions (e.g., Callaghan, 1989; Pashler, 1988), there is no possibility of checking whether segregation is performed in parallel or partially serially. Third, the present study (and Theeuwes, 1991) employed a task in which there is a clear separation between perceptual and response selection factors. Because the subjects responded to the orientation of the target line segment located in a perceptually discrepant display element, the task is what Duncan (1985) called a compound search task, in which the stimulus information separating target from nontargets tells nothing about which of the possible responses to choose. In this way, it is ensured that the RT data reflect effects operating at the early stage of perceptual processing rather than on processing operations occurring after the item has already been selected. For example, knowing the task-relevant stimulus feature might speed up the identification of an item that has already been selected, similarly to the way a prime speeds up processing of a target in a typical priming experiment. In other words, after entering the second stage of processing, less sensory evidence is required to decide whether an item is a target or a distractor (e.g., Broadbent's response set, 1970, 1982).

In short, the present study demonstrates that the parallel stage cannot selectively guide the attentive stage to just the known-to-be-relevant target feature. Because selectivity depends on the relative discriminability of each of the dimensions, the findings can be explained by a model that assumes that the preattentive stage calculates automatically differences in features within stimulus dimensions, followed by an attentive stage that automatically shifts to the location of the feature that pops out first.

\section{REFERENCES}

Broadbent, D. E. (1958). Perception and communication. London: Pergamon.

Broadbent, D. E. (1970). Stimulus and response set: Two kinds of selective attention. In D. Mostofsty (Ed.), Attention: Contemporary theories and analysis (pp. 51-60). New York: Appleton-Century-Crofts.

Broadbent, D. E. (1982). Task combination and the selective intake of information. Acta Psychologica, 50, 253-290. 
Bundesen, C. (1990). A theory of visual attention. Psychological Review, 97, 523-547.

Callaghan, T. C. (1989). Interference and dominance in texture segregation: Hue, geometric form, and line orientation. Perception \& Psychophysics, 46, 299-311.

Callaghan, T. C., Lasaga, M. I., \& Garner, W. R. (1986). Visual texture segregation based on orientation and hue. Perception \& Psychophysics, 39, 32-38

CAve, K. R., W Wolfe, J. M. (1990). Modeling the role of parallel processing in visual search. Cognitive Psychology, 22, 225-271.

Duncan, J. (1985). Visual search and visual attention. In M. Posner \& O. Marin (Eds.), Attention and performance $X I$ (pp. 85-106). Hillsdale, NJ: Erlbaum.

Duncan, J., \& Humphreys, G. W. (1989). Visual search and stimulus similarity. Psychological Review, 96, 433-458.

Neisser, U. (1967). Cognitive psychology. New York: AppletonCentury-Crofts.

Pashler, H. (1988). Cross-dimensional interaction and texture segregation. Perception \& Psychophysics, 43, 307-318.

SAGI, D., Julesz, B. (1985). "Where"' and "what" in vision. Science, 228, 1217-1219.
Shiffrin, R. M., Schneider, W. (1977). Controlled and automatic human information processing: II. Perceptual learning, automatic attending and a general theory. Psychological Review, 84, 127-190.

Theeuwes, J. (1991). Cross-dimensional perceptual selectivity. Perception \& Psychophysics, 50, 184-193.

Treisman, A. M. (1988). Feature and objects: The 14th Bartlett memorial lecture. Quarterly Journal of Experimental Psychology, 40, 201-237.

Treisman, A. M., \& Gelade, G. (1980). A feature integration theory of attention. Cognitive Psychology, 12, 97-136.

Treisman, A. M., Gormican, S. (1988). Feature search in early vision: Evidence from search asymmetries. Psychological Review, 95 , 15-48.

Treisman, A. M., SAto, S. (1990). Conjunction search revisited. Journal of Experimental Psychology: Human Perception \& Performance, 16, 459-478.

Ullman, S. (1984). Visual routines. Cognition, 18, 97-159.

(Manuscript received September 5, 1991; revision accepted for publication February 3, 1992.)

\section{Seventh International Conference on Event Perception and Action Vancouver, B.C., Canada August 8-13,1993}

The International Society for Ecological Psychology and the Psychology Department of the University of British Columbia are sponsoring the Seventh International Conference on Event Perception and Action to be held at the Conference Centre on the University of British Columbia campus.

This conference will present paper and poster sessions on a wide variety of topics, including, but not limited to, ecological acoustics, perceptual and cognitive development, perceptual guidance of locomotion, analysis of intentional systems, and ecological approaches to social psychology.

To have your name placed on the mailing list for the call for papers or for further information, contact John B. Pittenger, Department of Psychology, University of Arkansas at Little Rock, 2801 South University Avenue, Little Rock, AR 72204 (telephone 501-569-3171, e-mail icepa7@ualr.edu). 